Article

\title{
Some New Fixed Point Theorems in $b$-Metric Spaces with Application
}

\author{
Badriah A. S. Alamri ${ }^{1}$, Ravi P. Agarwal ${ }^{2,3}$ and Jamshaid Ahmad ${ }^{4, *}$ \\ 1 Department of Mathematics, King Abdulaziz University, P.O. Box 80203, Jeddah 21589, Saudi Arabia; \\ baalamri@kau.edu.sa \\ 2 Department of Mathematics, Texas A\&M University-Kingsville, 700 University Blvd, \\ Kingsville, TX 78363-8202, USA; ravi.agarwal@tamuk.edu \\ 3 Florida Institute of Technology, Melbourne, FL 32901, USA \\ 4 Department of Mathematics, University of Jeddah, P.O.Box 80327, Jeddah 21589, Saudi Arabia \\ * Correspondence: jkhan@uj.edu.sa
}

Received: 21 February 2020; Accepted: 13 April 2020; Published: 4 May 2020

\begin{abstract}
The aim of this article is to introduce a new class of contraction-like mappings, called the almost multivalued $\left(\Theta, \delta_{b}\right)$-contraction mappings in the setting of $b$-metric spaces to obtain some generalized fixed point theorems. As an application of our main result, we present the sufficient conditions for the existence of solutions of Fredholm integral inclusions. An example is also provided to verify the effectiveness and applicability of our main results.
\end{abstract}

Keywords: Fredholm integral inclusions; $\left(\Theta, \delta_{b}\right)$-contractions; $b$-metric space; fixed point; multivalued mappings

MSC: 46S40; 47H10; 54H25

\section{Introduction and Preliminaries}

The notion of metric space introduced by Frechet in 1906 is one of the pillars of not only mathematics but also physical sciences. Due to its importance and potential for application, this notion has been extended, improved and generalized in many different ways. The famous extensions of the concept of metric spaces has been done by Bakhtin [1], which was formally defined by Czerwik [2] in 1993 with a view of generalizing Banach contraction principle.

Definition 1. Let $\mathfrak{B}$ be a nonempty set and $s \geq 1$. A function $\sigma_{b}: \mathfrak{B} \times \mathfrak{B} \rightarrow \mathbb{R}_{0}^{+}$is said to be b-metric if these assertions hold:

$\left(b_{1}\right) \sigma_{b}(\varrho, \omega)=0 \Leftrightarrow \varrho=\omega$,

$\left(b_{2}\right) \sigma_{b}(\varrho, \omega)=\sigma_{b}(\omega, \varrho)$,

$\left(b_{3}\right) \sigma_{b}(\varrho, \omega) \leq s\left(\sigma_{b}(\varrho, \omega)+\sigma_{b}(\omega, \omega)\right)$

For all $\varrho, \mathfrak{\omega}, \omega \in \mathfrak{B}$. The triple $\left(\mathfrak{B}, \sigma_{b}, s\right)$ is called a $b$-metric space. Basic example of $b$-metric space which is not metric space is the following:

$\mathfrak{B}=\mathbb{R}$ and $\sigma_{b}: \mathfrak{B} \times \mathfrak{B} \rightarrow \mathbb{R}$ defined by

$$
\sigma_{b}(\varrho, \omega)=|\varrho-\omega|^{2}
$$

for all $\varrho, \mathfrak{\omega} \in \mathfrak{B}$ with $s=2$. 
Now, we supply a brief history for multivalued mappings defined in $\left(\mathfrak{B}, \sigma_{b}, s\right)$. Let $P_{b}(\mathfrak{B})$ and $P_{c b}(\mathfrak{B})$ represents the set of all bounded subsets and bounded and closed subsets of $\mathfrak{B}$ respectively. For $E_{1}, E_{2}, E_{3} \in P_{b}(\mathfrak{B})$, we define

$$
\sigma_{b}\left(E_{1}, E_{2}\right)=\inf \left\{\sigma_{b}(\varrho, \omega): \varrho \in E_{1}, \omega \in E_{2}\right\}
$$

and

$$
\delta_{b}\left(E_{1}, E_{2}\right)=\sup \left\{\sigma_{b}(\varrho, \omega): \varrho \in E_{1}, \omega \in E_{2}\right\}
$$

with

$$
\sigma_{b}\left(\varrho, E_{3}\right)=\sigma_{b}\left(\{\varrho\}, E_{3}\right)=\inf \left\{\sigma_{b}(\varrho, \omega): \varrho \in E_{1}, \omega \in E_{3}\right\}
$$

Now we review some simple properties of $\sigma_{b}$ and $\delta_{b}$ (see, e.g., [2-5]):

(i) If $E_{1}=\{\varrho\}$ and $E_{2}=\{\omega\}$, then $\sigma_{b}\left(E_{1}, E_{2}\right)=\delta_{b}\left(E_{1}, E_{2}\right)=\sigma_{b}(\varrho, \omega)$,

(ii) $\sigma_{b}\left(E_{1}, E_{2}\right) \leq \delta_{b}\left(E_{1}, E_{2}\right)$

(iii) $\sigma_{b}\left(\varrho, E_{2}\right) \leq \sigma_{b}(\varrho, \omega)$ for any $\omega \in E_{2}$,

(iv) $\delta_{b}\left(E_{1}, E_{3}\right) \leq s\left[\delta_{b}\left(E_{1}, E_{2}\right)+\delta_{b}\left(E_{2}, E_{3}\right)\right]$,

(v) $\delta_{b}\left(E_{1}, E_{2}\right)=0$ iff $E_{1}=E_{2}=\{\varrho\}$.

Furthermore, we will always assume that

(vi) $\sigma_{b}$ is continuous in its variables.

The notions of an orbit and orbitally continuous mapping presented in [6-8] for metric spaces can be generalized to the case of $b$-metric spaces, as follows:

Definition 2. Let $\left(\mathfrak{B}, \sigma_{b}, s\right)$ be a b-metric space and let $\mathcal{R}, \mathcal{R}_{1}, \mathcal{R}_{2}: \mathfrak{B} \rightarrow P_{b}(\mathfrak{B})$.

(1) An orbit $O\left(\varrho_{0}, \mathcal{R}\right)$ of $\mathcal{R}$ at $\varrho_{0}$ is any sequence $\left\{\varrho_{n}\right\}$ such that $\varrho_{n} \in \mathcal{R} \varrho_{n-1}$ for $n \in \mathbb{N}$.

(2) If for a point $\varrho_{0} \in \mathfrak{B}$, there exists a sequence $\left\{\varrho_{n}\right\}$ in $\mathfrak{B}$ such that $\varrho_{2 n+1} \in \mathcal{R}_{2} \varrho_{2 n}$ and $\varrho_{2 n+2} \in$ $\mathcal{R}_{1} \varrho_{2 n+1}$ for $n \in \mathbb{N} \cup\{0\}$, then $O\left(\varrho_{0}, \mathcal{R}_{1}, \mathcal{R}_{2}\right)=\left\{\varrho_{n}\right\}$ for $n \in \mathbb{N}$ is said to be an orbit of $\left(\mathcal{R}_{1}, \mathcal{R}_{2}\right)$ at $\varrho_{0}$.

(3) The space $\left(\mathfrak{B}, \sigma_{b}, s\right)$ is called $\left(\mathcal{R}_{1}, \mathcal{R}_{2}\right)$-orbitally complete if any Cauchy subsequence $\left\{\varrho_{n_{i}}\right\}$ of $O\left(\varrho_{0}, \mathcal{R}_{1}, \mathcal{R}_{2}\right)$ (for some $\varrho_{0}$ in $\mathfrak{B}$ ) converges in $\mathfrak{B}$. In particular, for $\mathcal{R}_{1}=\mathcal{R}_{2}=\mathcal{R}$, we say that $\mathfrak{B}$ is $\mathcal{R}$-orbitally complete.

(4) $\mathcal{R}$ is called an orbitally continuous at $\varrho_{0} \in \mathfrak{B}$ if for $\left\{\varrho_{n}\right\} \subset O\left(\varrho_{0}, \mathcal{R}\right)$ for $n \in \mathbb{N} \cup\{0\}$ and $\varrho^{*} \in \mathfrak{B}$, $\sigma_{b}\left(\varrho_{n}, \varrho^{*}\right) \rightarrow 0$ as $n \rightarrow \infty$ implies that $\delta_{b}\left(\mathcal{R} \varrho_{n}, \mathcal{R} \varrho^{*}\right) \rightarrow 0$ as $n \rightarrow \infty$.

(5) The graph $G(\mathcal{R})$ of $\mathcal{R}$ is defined as $G(\mathcal{R})=\{(\varrho, \omega): \varrho \in \mathfrak{B}, \boldsymbol{\omega} \in \mathcal{R} \varrho\}$. The graph $G(\mathcal{R})$ of $\mathcal{R}$ is said to be $\mathcal{R}$-orbitally closed if for $\left\{\varrho_{n}\right\}$, we get $\left(\varrho^{*}, \varrho^{*}\right) \in G(\mathcal{R})$ whenever $\left(\varrho_{n}, \varrho_{n+1}\right) \in G(\mathcal{R})$ and $\lim _{n \rightarrow \infty} \varrho_{n}=\varrho^{*}$.

In this paper, we define the notion of an almost multivalued $\left(\Theta, \delta_{b}\right)$-contraction and establish some new fixed point theorems in the context of $b$-metric spaces that generalize the main results of [9-11]. We also furnish a notable example to describe the significance of established results.

\section{Main Results}

Very recently, Jleli and Samet [9] gave a new variety of contractions, named as $\Theta$-contractions and obtained fixed point results for such contractions in the setting of generalized metric spaces. We review the existence of fixed points for multivalued mappings by adapting the ideas in [9] to the $b$-metric setting and inspired by Jleli et al. [9]. We give the following definition.

Definition 3. We represent by $\Omega_{s}(s \geq 1)$ the family of all functions $\Theta:(0, \infty) \rightarrow(1, \infty)$ satisfying the following properties: 
$\left(\Theta_{1}\right) \Theta$ is nondecreasing;

$\left(\Theta_{2}\right)$ for $\left\{\varrho_{n}\right\} \subseteq \mathbb{R}^{+}, \lim _{n \rightarrow \infty} \Theta\left(\varrho_{n}\right)=1 \Longleftrightarrow \lim _{n \rightarrow \infty}\left(\varrho_{n}\right)=0$;

$\left(\Theta_{3}\right) \exists 0<h<1$ and $l \in(0, \infty]$ such that $\lim _{\varrho \rightarrow 0^{+}} \frac{\Theta(\rho)-1}{\rho^{h}}=l$;

$\left(\Theta_{4}\right)$ for each sequence $\left\{\varrho_{n}\right\} \subseteq \mathbb{R}^{+}$such that $\Theta\left(s \varrho_{n}\right) \leq\left[\Theta\left(\varrho_{n-1}\right)\right]^{k}$ for all $n \in \mathbb{N}$ and some $k \in(0,1)$, then $\Theta\left(s_{n}^{n} \varrho\right) \leq\left[\Theta\left(s^{n-1} \varrho_{n-1}\right)\right]^{k}$ for all $n \in \mathbb{N}$.

Example 1. Let $\Theta:(0, \infty) \rightarrow(1, \infty)$ be defined by $\theta(\iota)=e^{\sqrt{l e^{\iota}}}$ for $\iota>0$. Clearly, $\Theta$ satisfies $\left(\Theta_{1}\right)-\left(\Theta_{4}\right)$. Here we show only $\left(\Theta_{4}\right)$. Assume that, for all $n \in \mathbb{N}$ and some $k \in(0,1)$, we have $\theta\left(\operatorname{se}_{n}\right) \leq\left[\theta\left(\varrho_{n-1}\right)\right]^{k}$, which implies that

$$
\begin{aligned}
e^{\sqrt{s \varrho_{n} e^{s \varrho_{n}}}} & \leq\left[e^{\sqrt{\varrho_{n-1} e^{\varrho_{n}-1}}}\right]^{k} \\
\sqrt{s \varrho_{n} e^{s \varrho_{n}}} & \leq k \sqrt{\varrho_{n-1} e^{\varrho_{n-1}}} .
\end{aligned}
$$

This implies that

$$
\sqrt{s \varrho_{n} e^{s \varrho_{n}-\varrho_{n-1}}} \leq k \sqrt{\varrho_{n-1}} .
$$

As $\theta\left(s \varrho_{n}\right) \leq\left[\theta\left(\varrho_{n-1}\right)\right]^{k} \leq \theta\left(\varrho_{n-1}\right)$. Also $\theta$ is nondecreasing, so $s \varrho_{n} \leq \varrho_{n-1}$ and $\rho_{n}-\varrho_{n-1} \leq 0$ implies $e^{s^{n-1}\left(s \varrho_{n}-\varrho_{n-1}\right)} \leq e^{s \varrho_{n}-\varrho_{n-1}}$. Therefore (1) implies

$$
\begin{aligned}
\sqrt{s \varrho_{n} e^{e^{n-1}\left(s \varrho_{n}-\varrho_{n-1}\right)}} & \leq k \sqrt{\varrho_{n-1}} \\
& \Longrightarrow \sqrt{\frac{s \varrho_{n} e^{s^{n} \varrho_{n}}}{e^{s^{-1} \varrho_{n-1}}}} \leq k \sqrt{\varrho_{n-1}} \\
& \Longrightarrow \sqrt{s \varrho_{n} e^{s^{n} \varrho_{n}}} \leq k \sqrt{\varrho_{n-1} e^{e^{n-1} \varrho_{n-1}}} \\
& \Longrightarrow \sqrt{s^{n} \varrho_{n} e^{s^{n} \varrho_{n}}} \leq k \sqrt{s^{n-1} \varrho_{n-1} e^{s^{n-1} \varrho_{n-1}}} \\
& \Longrightarrow e^{\sqrt{s^{n} \varrho_{n} e^{s^{n} \varrho_{n}}}} \leq e^{k \sqrt{s^{n-1} \varrho_{n-1} e^{s^{n-1} \varrho_{n-1}}}} \\
& \Longrightarrow \theta\left(s^{n} \varrho_{n}\right) \leq\left[\theta\left(s^{n-1} \varrho_{n-1}\right)\right]^{k}
\end{aligned}
$$

and hence $\left(\Theta_{4}\right)$ holds.

For more details regarding $\Theta$-contractions, we refer the reader to [10-19].

Definition 4. Let $\left(\mathfrak{B}, \sigma_{b}, s\right)$ be a b-metric space with coefficient $s \geq 1$. We say that $\mathcal{R}: \mathfrak{B} \rightarrow P_{b}(\mathfrak{B})$ is an almost multivalued $\left(\Theta, \delta_{b}\right)$-contraction if there exist some $\Theta \in \Omega_{s}, k \in(0,1)$ and $L \geq 0$ such that

$$
\Theta\left(s \delta_{b}(\mathcal{R} \varrho, \mathcal{R} \omega)\right) \leq\left[\Theta\left(m_{1}(\varrho, \omega)+\operatorname{Lm}_{2}(\varrho, \omega)\right)\right]^{k}
$$

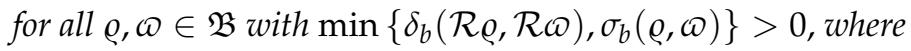

$$
m_{1}(\varrho, \omega)=\max \left\{\sigma_{b}(\varrho, \omega), \sigma_{b}(\varrho, \mathcal{R} \varrho), \sigma_{b}(\omega, \mathcal{R} \omega), \frac{\sigma_{b}(\varrho, \mathcal{R} \omega)+\sigma_{b}(\omega, \mathcal{R} \varrho)}{2 s}\right\}
$$

and

$$
m_{2}(\varrho, \omega)=\min \left\{\sigma_{b}(\varrho, \mathcal{R} \varrho), \sigma_{b}(\omega, \mathcal{R} \omega), \sigma_{b}(\varrho, \mathcal{R} \omega), \sigma_{b}(\omega, \mathcal{R} \varrho)\right\} .
$$

If (2) is satisfied just for $\varrho, \omega \in \overline{O\left(\varrho_{0}, \mathcal{R}\right)}$ (for some $\left.\varrho_{0} \in \mathfrak{B}\right)$, then $\mathcal{R}$ is said to be an almost multivalued orbitally $\left(\Theta, \delta_{b}\right)$-contraction.

Theorem 1. Let $\left(\mathfrak{B}, \sigma_{b}, s\right)$ be a b-metric space with coefficient $s>1$ and let $\mathcal{R}: \mathfrak{B} \rightarrow P_{b}(\mathfrak{B})$ an almost multivalued orbitally $\left(\Theta, \delta_{b}\right)$-contraction. Assume that $\left(\mathfrak{B}, \sigma_{b}, s\right)$ is $\mathcal{R}$-orbitally complete (for some $\left.\varrho_{0} \in \mathfrak{B}\right)$. 
If $\Theta$ is continuous and $\mathcal{R} \varrho$ is closed for all $\varrho \in \overline{O\left(\varrho_{0}, \mathcal{R}\right)}$ or $\mathcal{R}$ has $\mathcal{R}$-orbitally closed graph, then there exists $\varrho^{*} \in \mathfrak{B}$ such that $\varrho^{*} \in \mathcal{R} \varrho^{*}$.

Proof. For given $\varrho_{0} \in \mathfrak{B}$, we generate $\left\{\varrho_{n}\right\}$ in $\mathfrak{B}$ as $\varrho_{n+1} \in \mathcal{R} \varrho_{n}$, for all $n \geq 0$. If there exists $n_{0} \in \mathbb{N} \cup\{0\}$ for which $\varrho_{n_{0}}=\varrho_{n_{0}+1}$ then $\varrho_{n_{0}}$ is a fixed point of $\mathcal{R}$ and so the proof is finished. Thus, suppose that, for every $n \in \mathbb{N} \cup\{0\}, \varrho_{n} \neq \varrho_{n+1}$. So $\sigma_{b}\left(\varrho_{n+1}, \varrho_{n+2}\right)>0$ and $\delta_{b}\left(\mathcal{R} \varrho_{n}, \mathcal{R} \varrho_{n+1}\right)>0$ for all $n \geq 0$. Then, we have from (2) with the elements $\varrho=\varrho_{n}$ and $\mathscr{\omega}=\varrho_{n+1}$ that

$$
\Theta\left(s \sigma_{b}\left(\varrho_{n+1}, \varrho_{n+2}\right)\right) \leq \Theta\left(s \delta_{b}\left(\mathcal{R} \varrho_{n}, \mathcal{R} \varrho_{n+1}\right)\right) \leq\left[\Theta\left(m_{1}\left(\varrho_{n}, \varrho_{n+1}\right)+L m_{2}\left(\varrho_{n}, \varrho_{n+1}\right)\right)\right]^{k}
$$

where

$$
\begin{aligned}
m_{1}\left(\varrho_{n}, \varrho_{n+1}\right) & =\max \left\{\sigma_{b}\left(\varrho_{n}, \varrho_{n+1}\right), \sigma_{b}\left(\varrho_{n}, \mathcal{R} \varrho_{n}\right), \sigma_{b}\left(\varrho_{n+1}, \mathcal{R} \varrho_{n+1}\right), \frac{\sigma_{b}\left(\varrho_{n}, \mathcal{R} \varrho_{n+1}\right)+\sigma_{b}\left(\varrho_{n+1}, \mathcal{R} \varrho_{n}\right)}{2 s}\right\} \\
& \leq \max \left\{\sigma_{b}\left(\varrho_{n}, \varrho_{n+1}\right), \sigma_{b}\left(\varrho_{n}, \varrho_{n+1}\right), \sigma_{b}\left(\varrho_{n+1}, \varrho_{n+2}\right), \frac{1}{2 s} \sigma_{b}\left(\varrho_{n}, \varrho_{n+2}\right)\right\} \\
& =\max \left\{\sigma_{b}\left(\varrho_{n}, \varrho_{n+1}\right), \sigma_{b}\left(\varrho_{n+1}, \varrho_{n+2}\right), \frac{1}{2 s} \sigma_{b}\left(\varrho_{n}, \varrho_{n+2}\right)\right\}
\end{aligned}
$$

and

$$
m_{2}\left(\varrho_{n}, \varrho_{n+1}\right)=\min \left\{\sigma_{b}\left(\varrho_{n}, \mathcal{R} \varrho_{n}\right), \sigma_{b}\left(\varrho_{n+1}, \mathcal{R} \varrho_{n+1}\right), \sigma_{b}\left(\varrho_{n}, \mathcal{R} \varrho_{n+1}\right), \sigma_{b}\left(\varrho_{n+1}, \mathcal{R} \varrho_{n}\right)\right\}=0 .
$$

As $\frac{1}{2 s} \sigma_{b}\left(\varrho_{n}, \varrho_{n+2}\right) \leq \max \left\{\sigma_{b}\left(\varrho_{n}, \varrho_{n+1}\right), \sigma_{b}\left(\varrho_{n+1}, \varrho_{n+2}\right)\right\}$. So from (3), we have

$$
\Theta\left(s \sigma_{b}\left(\varrho_{n+1}, \varrho_{n+2}\right)\right) \leq\left[\Theta\left(\max \left\{\sigma_{b}\left(\varrho_{n}, \varrho_{n+1}\right), \sigma_{b}\left(\varrho_{n+1}, \varrho_{n+2}\right)\right\}\right)\right]^{k} .
$$

Assume that $\sigma_{b}\left(\varrho_{n}, \varrho_{n+1}\right) \leq \sigma_{b}\left(\varrho_{n+1}, \varrho_{n+2}\right)$, for some positive integer $n$. Then from (4), we have

$$
\Theta\left(s \sigma_{b}\left(\varrho_{n+1}, \varrho_{n+2}\right)\right) \leq\left[\Theta\left(\sigma_{b}\left(\varrho_{n+1}, \varrho_{n+2}\right)\right)\right]^{k} .
$$

a contradiction with $\left(\Theta_{1}\right)$. Hence,

$$
\max \left\{\sigma_{b}\left(\varrho_{n}, \varrho_{n+1}\right), \sigma_{b}\left(\varrho_{n+1}, \varrho_{n+2}\right)\right\}=\sigma_{b}\left(\varrho_{n}, \varrho_{n+1}\right)
$$

and consequently

$$
\Theta\left(s \sigma_{b}\left(\varrho_{n+1}, \varrho_{n+2}\right)\right) \leq\left[\Theta\left(\sigma_{b}\left(\varrho_{n}, \varrho_{n+1}\right)\right)\right]^{k}
$$

for all $n \in \mathbb{N} \cup\{0\}$. It follows by (5) and $\left(\Theta_{4}\right)$ that

$$
\Theta\left(s^{n} \sigma_{b}\left(\varrho_{n}, \varrho_{n+1}\right)\right) \leq\left[\Theta\left(s^{n-1} \sigma_{b}\left(\varrho_{n-1}, \varrho_{n}\right)\right)\right]^{k}
$$

for all $n \in \mathbb{N} \cup\{0\}$. Let us denote $\lambda_{n}=\sigma_{b}\left(\varrho_{n}, \varrho_{n+1}\right)$ for $n \in \mathbb{N} \cup\{0\}$. Then $\lambda_{n}>0$ for all $n$. Thus we have

$$
\Theta\left(s^{n} \lambda_{n}\right) \leq\left[\Theta\left(s^{n-1} \lambda_{n-1}\right)\right]^{k} \leq\left[\Theta\left(s^{n-2} \lambda_{n-2}\right)\right]^{k^{2}} \leq \cdots \leq\left[\Theta\left(\lambda_{0}\right)\right]^{k^{n}}
$$

for all $n \in \mathbb{N}$. Letting $n \rightarrow \infty$ in (7), we get

$$
\lim _{n \rightarrow \infty} \Theta\left(s^{n} \lambda_{n}\right)=1
$$

which implies that

$$
\lim _{n \rightarrow \infty} s^{n} \lambda_{n}=0
$$


by $\left(\Theta_{2}\right)$. By $\left(\Theta_{3}\right), \exists 0<h<1$ and $l \in(0, \infty]$ such that

$$
\lim _{n \rightarrow \infty} \frac{\Theta\left(s^{n} \lambda_{n}\right)-1}{\left(s^{n} \lambda_{n}\right)^{h}}=l
$$

Assume that $l<\infty$. For this case, let $q_{2}=\frac{l}{2}>0$. By the definition of the limit, $\exists n_{1} \in \mathbb{N}$ such that

$$
\left|\frac{\Theta\left(s^{n} \lambda_{n}\right)-1}{\left(s^{n} \lambda_{n}\right)^{h}}-l\right| \leq q_{2}
$$

for all $n>n_{1}$. This implies that

$$
\frac{\Theta\left(s^{n} \lambda_{n}\right)-1}{\left(s^{n} \lambda_{n}\right)^{h}} \geq l-q_{2}=\frac{l}{2}=q_{2}
$$

for all $n>n_{1}$. Then

$$
n\left(s^{n} \lambda_{n}\right)^{h} \leq q_{1} n\left[\Theta\left(s^{n} \lambda_{n}\right)-1\right]
$$

for all $n>n_{1}$, where $q_{1}=\frac{1}{q_{2}}$. Now assume that $l=\infty$. Let $q_{2}>0$. By the definition of the limit, there exists $n_{1} \in \mathbb{N}$ such that

$$
q_{2} \leq \frac{\Theta\left(s^{n} \lambda_{n}\right)-1}{\left(s^{n} \lambda_{n}\right)^{h}}
$$

for all $n>n_{1}$. This implies that

$$
n\left(s^{n} \lambda_{n}\right)^{h} \leq q_{1} n\left[\Theta\left(s^{n} \lambda_{n}\right)-1\right]
$$

for all $n>n_{1}$, where $q_{1}=\frac{1}{q_{2}}$. Thus, in all cases, there exists $q_{1}>0$ and $n_{1} \in \mathbb{N}$ such that

$$
n\left(s^{n} \lambda_{n}\right)^{h} \leq q_{1} n\left[\Theta\left(s^{n} \lambda_{n}\right)-1\right]
$$

for all $n>n_{1}$. Thus by (7) and (11), we get

$$
n\left(s^{n} \lambda_{n}\right)^{h} \leq q_{1} n\left(\left[\Theta\left(\lambda_{0}\right)\right]^{k^{n}}-1\right) .
$$

Letting $n \rightarrow \infty$ in (12), we get

$$
\lim _{n \rightarrow \infty} n\left(s^{n} \lambda_{n}\right)^{h}=0
$$

and hence $\lim _{n \rightarrow \infty} n^{\frac{1}{h}} s^{n} \lambda_{n}=0$ which yields that $\sum_{n=1}^{\infty} s^{n} \lambda_{n}$ is convergent. Thus $\left\{\varrho_{n}\right\}$ is a Cauchy sequence in $\overline{O\left(\varrho_{0}, \mathcal{R}\right)}$. As $\mathfrak{B}$ is $\mathcal{R}$-orbitally complete, there exists $\varrho^{*} \in \mathfrak{B}$ such that

$$
\varrho_{n} \rightarrow \varrho^{*} \text { as } n \rightarrow \infty \text {. }
$$

Assume that $\mathcal{R} \varrho^{*}$ is closed. We see that if there exists $\left\{n_{k}\right\} \subset \mathbb{N}$ such that $\varrho_{n_{k}} \in \mathcal{R} \varrho^{*}, \forall k \in \mathbb{N}$. As $\mathcal{R} \varrho^{*}$ is closed and $\lim _{k \rightarrow \infty} \varrho_{n_{k}}=\varrho^{*}$, so we conclude that $\varrho^{*} \in \mathcal{R} \varrho^{*}$. Thus the proof is finished. Then we suppose that there exists $n_{0} \in \mathbb{N}$ such that $\varrho_{n} \notin \mathcal{R} \varrho^{*}, \forall n \in \mathbb{N}$ with $n \geq n_{0}$. It follows that $\delta_{b}\left(\mathcal{R} \varrho_{n}, \mathcal{R} \varrho^{*}\right)>0, \forall n \geq n_{0}$. Then, we have from (2) with the elements $\varrho=\varrho_{n}$ and $\boldsymbol{\omega}=\varrho^{*}$ that

$$
\Theta\left(s \sigma_{b}\left(\varrho_{n+1}, \mathcal{R} \varrho^{*}\right)\right)=\Theta\left(s \delta_{b}\left(\mathcal{R} \varrho_{n}, \mathcal{R} \varrho^{*}\right)\right) \leq\left[\Theta\left(m_{1}\left(\varrho_{n}, \varrho^{*}\right)+L m_{2}\left(\varrho_{n}, \varrho^{*}\right)\right)\right]^{k}
$$

where

$$
\begin{aligned}
m_{1}\left(\varrho_{n}, \varrho^{*}\right) & =\max \left\{\sigma_{b}\left(\varrho_{n}, \varrho^{*}\right), \sigma_{b}\left(\varrho_{n}, \mathcal{R} \varrho_{n}\right), \sigma_{b}\left(\varrho^{*}, \mathcal{R} \varrho^{*}\right), \frac{\sigma_{b}\left(\varrho_{n}, \mathcal{R} \varrho^{*}\right)+\sigma_{b}\left(\varrho^{*}, \mathcal{R} \varrho_{n}\right)}{2 s}\right\} \\
& \leq \max \left\{\sigma_{b}\left(\varrho_{n}, \varrho^{*}\right), \sigma_{b}\left(\varrho_{n}, \varrho_{n+1}\right), \sigma_{b}\left(\varrho^{*}, \mathcal{R} \varrho^{*}\right), \frac{\sigma_{b}\left(\varrho_{n}, \mathcal{R} \varrho^{*}\right)+\sigma_{b}\left(\varrho^{*}, \varrho_{n+1}\right)}{2 s}\right\} \\
& \rightarrow \sigma_{b}\left(\varrho^{*}, \mathcal{R} \varrho^{*}\right) \text { as } n \rightarrow \infty .
\end{aligned}
$$


and

$$
\begin{aligned}
m_{2}\left(\varrho_{n}, \varrho^{*}\right) & =\min \left\{\sigma_{b}\left(\varrho_{n}, \mathcal{R} \varrho_{n}\right), \sigma_{b}\left(\varrho^{*}, \mathcal{R} \varrho^{*}\right), \sigma_{b}\left(\varrho_{n}, \mathcal{R} \varrho^{*}\right), \sigma_{b}\left(\varrho^{*}, \mathcal{R} \varrho_{n}\right)\right\} \\
& \leq \min \left\{\sigma_{b}\left(\varrho_{n}, \varrho_{n+1}\right), \sigma_{b}\left(\varrho^{*}, \mathcal{R} \varrho^{*}\right), \sigma_{b}\left(\varrho_{n}, \mathcal{R} \varrho^{*}\right), \sigma_{b}\left(\varrho^{*}, \varrho_{n+1}\right)\right\} \\
& \rightarrow 0 \text { as } n \rightarrow \infty
\end{aligned}
$$

Since $\Theta$ and $\sigma_{b}$ are continuous, so taking the limit of (15) as $n \rightarrow \infty$, we have

$$
\Theta\left(s \sigma_{b}\left(\varrho^{*}, \mathcal{R} \varrho^{*}\right)\right) \leq\left[\Theta\left(\sigma_{b}\left(\varrho^{*}, \mathcal{R} \varrho^{*}\right)\right]^{k}\right.
$$

which is impossible because $k \in(0,1)$ and $s \geq 1$. Since $\Theta$ is strictly increasing. Hence, $\sigma_{b}\left(\varrho^{*}, \mathcal{R} \varrho^{*}\right)=0$ and as and, since $\mathcal{R} \varrho^{*}$ is closed, we have $\varrho^{*} \in \mathcal{R} \varrho^{*}$. Thus, $\varrho^{*}$ is a fixed point of $\mathcal{R}$. Assume that $G(\mathcal{R})$ is $\mathcal{R}$-orbitally closed and since $\left(\varrho_{n}, \varrho_{n+1}\right) \in G(\mathcal{R})$ for all $n \in \mathbb{N} \cup\{0\}$ and $\lim _{n \rightarrow \infty} \varrho_{n}=\varrho^{*}$, we have $\left(\varrho^{*}, \varrho^{*}\right) \in G(\mathcal{R})$ by the $\mathcal{R}$-orbitally closedness. Hence $\varrho^{*} \in \mathcal{R} \varrho^{*}$.

The following corollary follows from Theorem 1 by taking $\Theta(\iota)=e^{\sqrt{\iota}}$ for $\iota>0$.

Corollary 1. Let $\left(\mathfrak{B}, \sigma_{b}, s\right)$ be a b-metric space with coefficient $s \geq 1$ and let $\mathcal{R}: \mathfrak{B} \rightarrow P_{b}(\mathfrak{B})$ satisfying, for some $k \in(0,1), \varrho_{0} \in \mathfrak{B}$ and $L \geq 0$ such that

$$
s \delta_{b}(\mathcal{R} \varrho, \mathcal{R} \omega) \leq k\left(m_{1}(\varrho, \omega)+\operatorname{Lm}_{2}(\varrho, \omega)\right)
$$

for all $\varrho, \omega \in \mathfrak{B}$ with $\min \left\{\delta_{b}(\mathcal{R} \varrho, \mathcal{R} \omega), \sigma_{b}(\varrho, \omega)\right\}>0$, where

$$
m_{1}(\varrho, \omega)=\max \left\{\sigma_{b}(\varrho, \omega), \sigma_{b}(\varrho, \mathcal{R} \varrho), \sigma_{b}(\omega, \mathcal{R} \omega), \frac{\sigma_{b}(\varrho, \mathcal{R} \omega)+\sigma_{b}(\omega, \mathcal{R} \varrho)}{2 s}\right\}
$$

and

$$
m_{2}(\varrho, \omega)=\min \left\{\sigma_{b}(\varrho, \mathcal{R} \varrho), \sigma_{b}(\omega, \mathcal{R} \omega), \sigma_{b}(\varrho, \mathcal{R} \omega), \sigma_{b}(\omega, \mathcal{R} \varrho)\right\}
$$

for all $\varrho, \omega \in \overline{O\left(\varrho_{0}, \mathcal{R}\right)}$. Assume that $\left(\mathfrak{B}, \sigma_{b}, s\right)$ is $\mathcal{R}$-orbitally complete (for some $\left.\varrho_{0} \in \mathfrak{B}\right)$. If $\mathcal{R} \varrho$ is closed, $\forall$ $\varrho \in \overline{O\left(\varrho_{0}, \mathcal{R}\right)}$ or $\mathcal{R}$ has $\mathcal{R}$-orbitally closed graph, then there exists $\varrho^{*} \in \mathfrak{B}$ such that $\varrho^{*} \in \mathcal{R} \varrho^{*}$.

Example 2. Let $\mathfrak{B}=[0,1]$ be endowed with b-metric

$$
\sigma_{b}(\varrho, \omega)=(\varrho-\omega)^{2}
$$

with coefficient $s=2$. Define the mapping $\mathcal{R}: \mathfrak{B} \rightarrow P_{c b}(\mathfrak{B})$ given by

$$
\mathcal{R} \varrho=\left\{\begin{array}{c}
\left\{\frac{1}{3}\right\}, 0 \leq \varrho<1 \\
{\left[0, \frac{1}{4}\right], \varrho=1}
\end{array}\right.
$$

If $\varrho, \omega \in[0,1)$, then $\delta_{b}(\mathcal{R} \varrho, \mathcal{R} \omega)=0$. Let $\varrho \in[0,1)$ and $\omega=1$. Then $\mathcal{R} \varrho=\left\{\frac{1}{3}\right\}, \mathcal{R} \omega=\left[0, \frac{1}{4}\right]$ and $\delta_{b}(\mathcal{R} \varrho, \mathcal{R} \omega)=\frac{1}{12}$,

$$
\begin{gathered}
m_{1}(\varrho, \omega)=\max \left\{(1-\varrho)^{2},\left(\frac{1}{3}-\varrho\right)^{2},\left(\frac{3}{4}\right)^{2}, \frac{1}{4}\left[\sigma_{b}(\varrho, \mathcal{R} \omega)+\left(\frac{2}{3}\right)^{2}\right]\right\} \geq \frac{9}{16} \\
m_{2}(\varrho, \omega)=\min \left\{\left(\frac{1}{3}-\varrho\right)^{2},\left(\frac{3}{4}\right)^{2}, \sigma_{b}(\varrho, \mathcal{R} \omega),\left(\frac{2}{3}\right)^{2}\right\} \geq 0 .
\end{gathered}
$$


Take $k=\frac{2}{9}, \Theta(\iota)=e^{\sqrt{\iota}}$ and $L \geq 0$. Then

$$
\begin{aligned}
\Theta\left(s \delta_{b}(\mathcal{R} \varrho, \mathcal{R} \omega)\right) & =\Theta\left(2 \cdot \frac{1}{12}\right)=e^{\sqrt{\frac{1}{6}}} \\
& <e^{\frac{2}{3}}=e^{\frac{8}{9} \cdot \frac{3}{4}} \\
& =\left[\Theta\left(m_{1}(\varrho, \omega)+\operatorname{Lm} m_{2}(\varrho, \omega)\right)\right]^{k} .
\end{aligned}
$$

Hence, the assertions of Theorem 1 are fulfilled and $\mathcal{R}$ has a fixed point (which is $\varrho^{*}=\frac{1}{3}$ ).

The family $\Omega_{S}$ consists of a broad set of functions. For example, if we take

$$
\Theta(\iota)=2-\frac{2}{\pi} \arctan \left(\frac{1}{\iota^{\beta}}\right)
$$

where $0<\beta<1$ and $\iota>0$, we can obatin the following result from our main Theorem 1 .

Corollary 2. Let $\left(\mathfrak{B}, \sigma_{b}, s\right)$ be a b-metric space with coefficient $s \geq 1$ and let $\mathcal{R}: \mathfrak{B} \rightarrow P_{b}(\mathfrak{B})$ satisfying, for some $k, \beta \in(0,1), \varrho_{0} \in \mathfrak{B}$ and $L \geq 0$ such that

$$
2-\frac{2}{\pi} \arctan \left(\frac{1}{\left(s \delta_{b}(\mathcal{R} \varrho, \mathcal{R} \omega)\right)^{\beta}}\right) \leq\left[2-\frac{2}{\pi} \arctan \left(\frac{1}{\left(m_{1}(\varrho, \omega)+L m_{2}(\varrho, \omega)\right)^{\beta}}\right)\right]^{k}
$$

for all $\varrho, \omega \in \mathfrak{B}$ with $\min \left\{\delta_{b}(\mathcal{R} \varrho, \mathcal{R} \omega), \sigma_{b}(\varrho, \omega)\right\}>0$, where

$$
m_{1}(\varrho, \omega)=\max \left\{\sigma_{b}(\varrho, \omega), \sigma_{b}(\varrho, \mathcal{R} \varrho), \sigma_{b}(\omega, \mathcal{R} \omega), \frac{\sigma_{b}(\varrho, \mathcal{R} \omega)+\sigma_{b}(\omega, \mathcal{R} \varrho)}{2 s}\right\}
$$

and

$$
m_{2}(\varrho, \omega)=\min \left\{\sigma_{b}(\varrho, \mathcal{R} \varrho), \sigma_{b}(\omega, \mathcal{R} \omega), \sigma_{b}(\varrho, \mathcal{R} \omega), \sigma_{b}(\omega, \mathcal{R} \varrho)\right\}
$$

for all $\varrho, \omega \in \overline{O\left(\varrho_{0}, \mathcal{R}\right)}$. Suppose that $\left(\mathfrak{B}, \sigma_{b}, s\right)$ is $\mathcal{R}$-orbitally complete (for some $\left.\varrho_{0} \in \mathfrak{B}\right)$. If $\mathcal{R} \varrho$ is closed, $\forall$ $\varrho \in \overline{O\left(\varrho_{0}, \mathcal{R}\right)}$ or $\mathcal{R}$ has $\mathcal{R}$-orbitally closed graph, then there exists $\varrho^{*} \in \mathfrak{B}$ such that $\varrho^{*} \in \mathcal{R} \varrho^{*}$.

Now we give some results for $\mathcal{R}: \mathfrak{B} \rightarrow \mathfrak{B}$ as the consequences of Theorem 1 .

Corollary 3. Let $\left(\mathfrak{B}, \sigma_{b}, s\right)$ be a b-metric space with coefficient $s>1$ and let $\mathcal{R}: \mathfrak{B} \rightarrow \mathfrak{B}$ such that $\mathfrak{B}$ is $\mathcal{R}$-orbitally complete (at some $\varrho_{0}$ ). Assume that there exists some $\Theta \in \Omega_{s}, k \in(0,1)$ and $L \geq 0$ such that

$$
\Theta\left(s \sigma_{b}(\mathcal{R} \varrho, \mathcal{R} \omega)\right) \leq\left[\Theta\left(m_{1}^{\prime}(\varrho, \omega)+L m_{2}^{\prime}(\varrho, \omega)\right)\right]^{k}
$$

for all $\varrho, \omega \in \mathfrak{B}$ with $\min \left\{\sigma_{b}(\mathcal{R} \varrho, \mathcal{R} \omega), \sigma_{b}(\varrho, \omega)\right\}>0$, where

$$
m_{1}^{\prime}(\varrho, \omega)=\max \left\{\sigma_{b}(\varrho, \omega), \sigma_{b}(\varrho, \mathcal{R} \varrho), \sigma_{b}(\omega, \mathcal{R} \omega), \frac{\sigma_{b}(\varrho, \mathcal{R} \omega)+\sigma_{b}(\omega, \mathcal{R} \varrho)}{2 s}\right\}
$$

and

$$
m_{2}^{\prime}(\varrho, \omega)=\min \left\{\sigma_{b}(\varrho, \mathcal{R} \varrho), \sigma_{b}(\omega, \mathcal{R} \omega), \sigma_{b}(\varrho, \mathcal{R} \omega), \sigma_{b}(\omega, \mathcal{R} \varrho)\right\} .
$$

If $\Theta$ is continuous, then $\mathcal{R}$ has a fixed point in $\mathfrak{B}$.

The following result is an extension and generalization of the main result of [10] with the consideration of an orbitally complete $b$-metric space. 
Corollary 4 . Let $\left(\mathfrak{B}, \sigma_{b}, s\right)$ be a b-metric space with coefficient $s \geq 1$ and let $\mathcal{R}: \mathfrak{B} \rightarrow \mathfrak{B}$ such that $\mathfrak{B}$ is $\mathcal{R}$-orbitally complete (at some $\varrho_{0}$ ). Assume that there exists some $\Theta \in \Omega_{s}, k \in(0,1)$ such that

$$
\Theta\left(s \sigma_{b}(\mathcal{R} \varrho, \mathcal{R} \omega)\right) \leq\left[\Theta\left(m_{1}^{\prime}(\varrho, \omega)\right)\right]^{k}
$$

for all $\varrho, \omega \in \mathfrak{B}$ with $\min \left\{\sigma_{b}(\mathcal{R} \varrho, \mathcal{R} \omega), \sigma_{b}(\varrho, \omega)\right\}>0$, where

$$
m_{1}^{\prime}(\varrho, \omega)=\max \left\{\sigma_{b}(\varrho, \omega), \sigma_{b}(\varrho, \mathcal{R} \varrho), \sigma_{b}(\omega, \mathcal{R} \omega), \frac{\sigma_{b}(\varrho, \mathcal{R} \omega)+\sigma_{b}(\omega, \mathcal{R} \varrho)}{2 s}\right\}
$$

If $\Theta$ is continuous, then $\mathcal{R}$ has a fixed point in $\mathfrak{B}$.

Remark 1. Corollary 4 is itself an extension of the main result of [9] in orbitally complete b-metric space.

Remark 2. One can easily derive the main result of [11] by taking $s=1$ in Corollary 4 in this way.

\section{Applications}

The purpose of this section is to prove the existence of solutions for a Fredholm-type integral inclusion

$$
\varrho(\iota) \in f(\iota)+\int_{a}^{b} K(\iota, \varsigma, \varrho(\varsigma)) \sigma \zeta, \quad \iota \in[a, b]
$$

where $K:[a, b] \times[a, b] \times \mathbb{R} \rightarrow P_{c b}(\mathbb{R})$ a given multivalued operator, $f \in C[a, b]$ is a given real-valued function and $\varrho \in C[a, b]$ is the unknown function.

Consider $\sigma_{b}$ on $C[a, b]$ defined by

$$
\sigma_{b}(\varrho, \omega)=\left(\max _{\iota \in[a, b]}|\varrho(\iota)-\omega(\iota)|\right)^{p}=\max _{\iota \in[a, b]}|\varrho(\iota)-\omega(\iota)|^{p}
$$

for all $\varrho, \omega \in C[a, b]$ and for $p \geq 1$. Then $\left(C[a, b], \sigma_{b}, 2^{p-1}\right)$ is a complete $b$-metric space.

Suppose the following assertions hold.

(a) for all $\varrho \in C[a, b]$, the operator $K_{\varrho}(l, \varsigma)=K(\iota, \zeta, \varrho(\varsigma))$, where $\iota, \varsigma \in[a, b]$ is continuous.

(b) there exists $Y:[a, b] \times[a, b] \rightarrow[0,+\infty)$ such that

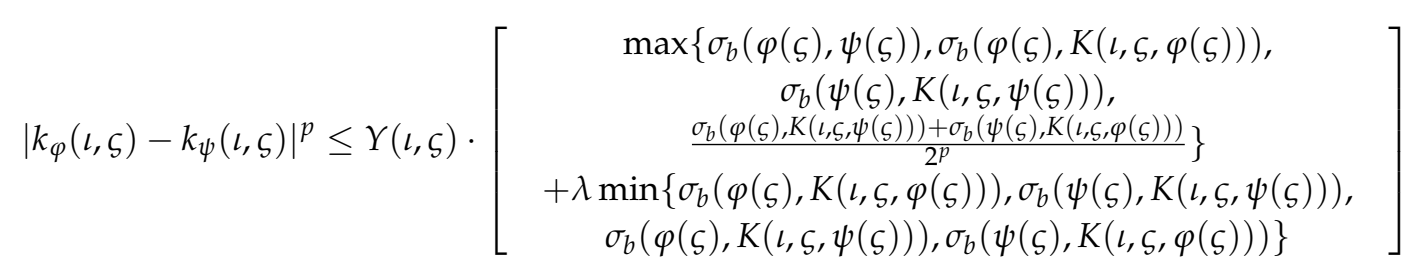

for all $\iota, \varsigma \in[a, b], \varphi, \psi \in C[a, b]$ and all $k_{\varphi}(\iota, \varsigma) \in K_{\varphi}(\iota, \varsigma), k_{\psi}(\iota, \varsigma) \in K_{\psi}(l, \varsigma)$, where $\lambda \geq 0$ and $p>1$.

(c) there exists $k \in(0,1)$ such that

$$
\sup _{l \in[a, b]} \int_{a}^{b} Y(l, \varsigma) \sigma \varsigma \leq \frac{k}{2^{p-1}}
$$

Theorem 2. With the assertions (a)-(c), the integral inclusion (16) has a solution in $C[a, b]$.

Proof. Let $\mathfrak{B}=C[a, b]$ (with b-metric $\sigma_{b}$ as defined in (17) and consider the multivalued operator $\mathcal{R}: \mathfrak{B} \rightarrow P_{c b}(\mathfrak{B})$ defined by

$$
\mathcal{R} \varrho=\left\{\omega \in \mathfrak{B}: \omega(\iota) \in f(\iota)+\int_{a}^{b} K(\iota, \varsigma, \varrho(\varsigma)) \sigma \varsigma, \iota \in[a, b]\right\}
$$


Let $\varrho \in \mathfrak{B}$. For $K_{\varrho}(l, \zeta):[a, b] \times[a, b] \rightarrow P_{c b}(\mathbb{R}), \exists k_{\varrho}(l, \zeta):[a, b] \times[a, b] \rightarrow \mathbb{R}$ such that $k_{\varrho}(l, \zeta) \in K_{\varrho}(l, \zeta)$ for each $\iota, \varsigma \in[a, b]$. This implies that $f(\iota)+\int_{a}^{b} k_{\varrho}(\iota, \varsigma) \sigma \varsigma \in \mathcal{R} \varrho$. Hence $\mathcal{R} \varrho \neq \varnothing$. As Since $f \in C([a, b])$ and $K_{\varrho}(l, \varsigma)$ is continuous on $[a, b] \times[a, b]$, so their ranges are bounded. It follows that $\mathcal{R} \varrho$ is also bounded. Now we prove that (2) holds for $\mathcal{R}$ in $\mathfrak{B}$ with some $k \in(0,1), L \geq 0$ and $\Theta \in \Omega_{s}$, i.e.,

$$
\Theta\left(s \delta_{b}\left(\mathcal{R} \varrho_{1}, \mathcal{R} \varrho_{2}\right)\right) \leq\left[\Theta\left(\begin{array}{c}
\max \left\{\sigma_{b}\left(\varrho_{1}, \varrho_{2}\right), \sigma_{b}\left(\varrho_{1}, \mathcal{R} \varrho_{1}\right), \sigma_{b}\left(\varrho_{2}, \mathcal{R} \varrho_{2}\right), \frac{\sigma_{b}\left(\varrho_{1}, \mathcal{R} \varrho_{2}\right)+\sigma_{b}\left(\varrho_{2}, \mathcal{R} \varrho_{1}\right)}{2^{p}}\right\} \\
+L \min \left\{\sigma_{b}\left(\varrho_{1}, \mathcal{R} \varrho_{1}\right), \sigma_{b}\left(\varrho_{2}, \mathcal{R} \varrho_{2}\right), \sigma_{b}\left(\varrho_{1}, \mathcal{R} \varrho_{2}\right), \sigma_{b}\left(\varrho_{2}, \mathcal{R} \varrho_{1}\right)\right\}
\end{array}\right)\right]^{k}
$$

for elements $\varrho_{1}, \varrho_{2} \in \mathfrak{B}$. Let $\omega_{1} \in \mathcal{R} \varrho_{1}$ be arbitrary, i.e.,

$$
\omega_{1}(\iota) \in f(\iota)+\int_{a}^{b} K\left(\iota, \varsigma, \varrho_{1}(\varsigma)\right) \sigma \varsigma
$$

for $\iota \in[a, b]$ holds. It means that $\exists k_{\varrho_{1}}(\iota, \varsigma) \in K_{\varrho_{1}}(\iota, \varsigma)=K\left(\iota, \varsigma, \varrho_{1}(\varsigma)\right)$ such that

$$
\omega_{1}(\iota)=f(\iota)+\int_{a}^{b} k_{\varrho_{1}}(\iota, \varsigma) \sigma \varsigma
$$

for $\iota, \varsigma \in[a, b]$. Now for all $\varrho_{1}, \varrho_{2} \in \mathfrak{B}$, it follows from (b) that

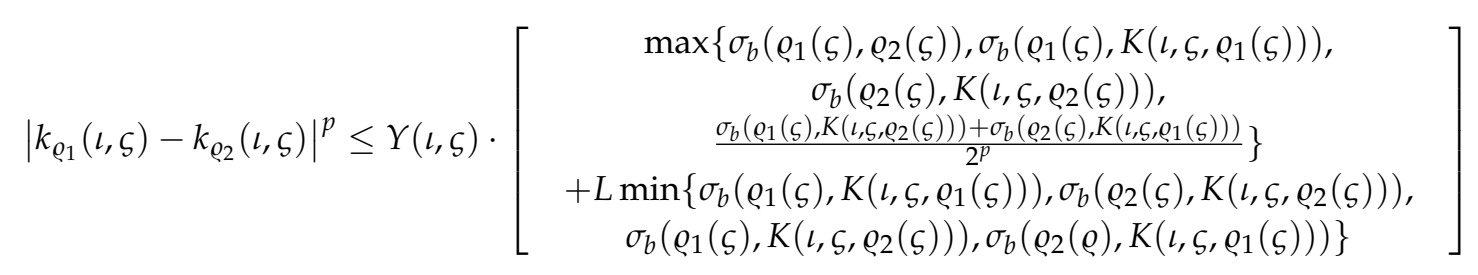

This means that there exists $\omega(l, \zeta) \in K_{\varrho_{2}}(l, \zeta)$ such that

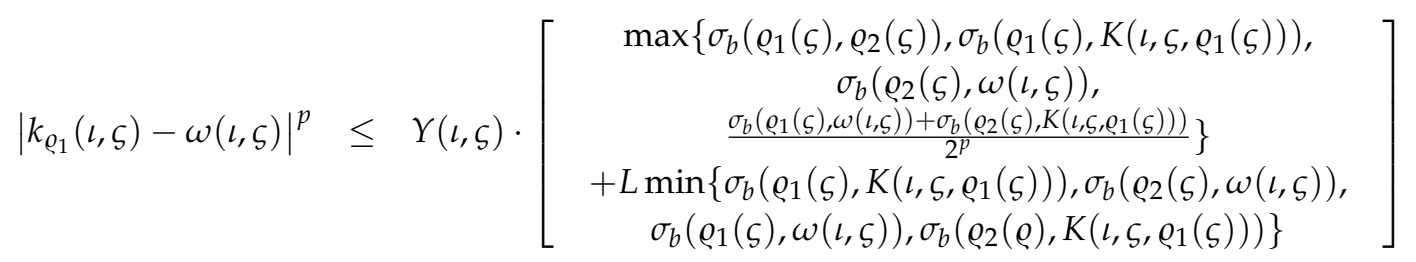

$$
\begin{aligned}
& =R(\iota, \zeta) \text {. }
\end{aligned}
$$

for all $\iota, \varsigma \in[a, b]$. We represent the operator $U(\iota, \varsigma):[a, b] \times[a, b] \rightarrow P_{c b}(\mathbb{R})$ by

$$
U(l, \varsigma)=K_{\varrho_{2}}(l, \varsigma) \cap\left\{\varphi \in \mathbb{R}: \sigma_{b}\left(k_{\varrho_{1}}(l, \varsigma), \varphi\right) \leq R(l, \varsigma)\right\} .
$$

Hence, by (a), $U$ is lower semicontinuous, this implies that $\exists k_{\varrho_{2}}(l, \zeta):[a, b] \times[a, b] \rightarrow \mathbb{R}$ such that $k_{\varrho_{2}}(l, \varsigma) \in U(\iota, \varsigma), \forall \iota, \varsigma \in[a, b]$. Then $\omega_{2}(\iota)=f(\iota)+\int_{a}^{b} k_{\varrho_{1}}(\iota, \varsigma) \sigma \varsigma$ satisfies that

$$
\omega_{1}(\iota) \in f(\iota)+\int_{a}^{b} K\left(\iota, \zeta, \varrho_{1}(\zeta)\right) \sigma \zeta, \quad \iota \in[a, b]
$$

$\iota \in[a, b]$. That is $\omega_{2} \in \mathcal{R} \varrho_{2}$ and

$$
\sigma_{b}\left(\omega_{1}, \omega_{2}\right) \leq \max _{\iota \in[a, b]} \int_{a}^{b}\left|k_{\varrho_{1}}(l, \zeta)-k_{\varrho_{2}}(\iota, \zeta)\right|^{p} \sigma \zeta
$$




$$
\begin{aligned}
& \leq \max _{\iota \in[a, b]} \int_{a}^{b} Y(\iota, \varsigma) \cdot\left[\begin{array}{c}
\max \left\{\sigma_{b}\left(\varrho_{1}(\varsigma), \varrho_{2}(\varsigma)\right), \sigma_{b}\left(\varrho_{1}(\varsigma), K\left(l, \varsigma, \varrho_{1}(\varsigma)\right)\right),\right. \\
\sigma_{b}\left(\varrho_{2}(\varsigma), K\left(l, \varsigma, \varrho_{2}(\varsigma)\right)\right), \\
\left.\frac{\sigma_{b}\left(\varrho_{1}(\varsigma), K\left(l, \zeta, \varrho_{2}(\varsigma)\right)\right)+\sigma_{b}\left(\varrho_{2}(\varsigma), K\left(l, \zeta, \varrho_{1}(\varsigma)\right)\right)}{2^{p}}\right\} \\
+L \min \left\{\sigma_{b}\left(\varrho_{1}(\varsigma), K\left(l, \varsigma, \varrho_{1}(\varsigma)\right)\right), \sigma_{b}\left(\varrho_{2}(\varsigma), K\left(\iota, \varsigma, \varrho_{2}(\varsigma)\right)\right),\right. \\
\left.\sigma_{b}\left(\varrho_{1}(\varsigma), K\left(l, \zeta, \varrho_{2}(\varsigma)\right)\right), \sigma_{b}\left(\varrho_{2}(\varrho), K\left(l, \varsigma, \varrho_{1}(\varsigma)\right)\right)\right\}
\end{array}\right] \sigma \varsigma \\
& \leq \frac{k}{2^{p-1}} \cdot\left[\begin{array}{c}
\max \left\{\sigma_{b}\left(\varrho_{1}, \varrho_{2}\right), \sigma_{b}\left(\varrho_{1}, \mathcal{R} \varrho_{1}\right), \sigma_{b}\left(\varrho_{2}, \mathcal{R} \varrho_{2}\right), \frac{\sigma_{b}\left(\varrho_{1}, \mathcal{R} \varrho_{2}\right)+\sigma_{b}\left(\varrho_{2}, \mathcal{R} \varrho_{1}\right)}{2^{p}}\right\} \\
+L \min \left\{\sigma_{b}\left(\varrho_{1}, \mathcal{R} \varrho_{1}\right), \sigma_{b}\left(\varrho_{2}, \mathcal{R} \varrho_{2}\right), \sigma_{b}\left(\varrho_{1}, \mathcal{R} \varrho_{2}\right), \sigma_{b}\left(\varrho_{2}, \mathcal{R} \varrho_{1}\right)\right\}
\end{array}\right]
\end{aligned}
$$

for all $\iota, \varsigma \in[a, b]$. Thus, we get

$$
\begin{gathered}
\delta_{b}\left(\mathcal{R} \varrho_{1}, \mathcal{R} \varrho_{2}\right) \leq \frac{k}{2^{p-1}} \cdot\left[\begin{array}{c}
\max \left\{\sigma_{b}\left(\varrho_{1}, \varrho_{2}\right), \sigma_{b}\left(\varrho_{1}, \mathcal{R} \varrho_{1}\right), \sigma_{b}\left(\varrho_{2}, \mathcal{R} \varrho_{2}\right), \frac{\sigma_{b}\left(\varrho_{1}, \mathcal{R} \varrho_{2}\right)+\sigma_{b}\left(\varrho_{2}, \mathcal{R} \varrho_{1}\right)}{2^{p}}\right\} \\
+L \min \left\{\sigma_{b}\left(\varrho_{1}, \mathcal{R} \varrho_{1}\right), \sigma_{b}\left(\varrho_{2}, \mathcal{R} \varrho_{2}\right), \sigma_{b}\left(\varrho_{1}, \mathcal{R} \varrho_{2}\right), \sigma_{b}\left(\varrho_{2}, \mathcal{R} \varrho_{1}\right)\right\}
\end{array}\right] \\
s \delta_{b}\left(\mathcal{R} \varrho_{1}, \mathcal{R} \varrho_{2}\right) \leq k\left[\begin{array}{c}
\max \left\{\sigma_{b}\left(\varrho_{1}, \varrho_{2}\right), \sigma_{b}\left(\varrho_{1}, \mathcal{R} \varrho_{1}\right), \sigma_{b}\left(\varrho_{2}, \mathcal{R} \varrho_{2}\right), \frac{\sigma_{b}\left(\varrho_{1}, \mathcal{R} \varrho_{2}\right)+\sigma_{b}\left(\varrho_{2}, \mathcal{R} \varrho_{1}\right)}{2^{p}}\right\} \\
+L \min \left\{\sigma_{b}\left(\varrho_{1}, \mathcal{R} \varrho_{1}\right), \sigma_{b}\left(\varrho_{2}, \mathcal{R} \varrho_{2}\right), \sigma_{b}\left(\varrho_{1}, \mathcal{R} \varrho_{2}\right), \sigma_{b}\left(\varrho_{2}, \mathcal{R} \varrho_{1}\right)\right\}
\end{array}\right] .
\end{gathered}
$$

Taking exponential on both side, we have

$$
e^{s \delta_{b}\left(\mathcal{R} \varrho_{1}, \mathcal{R} \varrho_{2}\right)} \delta_{b}\left(\mathcal{R} \varrho_{1}, \mathcal{R} \varrho_{2}\right) \leq e^{k\left[\begin{array}{c}
\max \left\{\sigma_{b}\left(\varrho_{1}, \varrho_{2}\right), \sigma_{b}\left(\varrho_{1}, \mathcal{R} \varrho_{1}\right), \sigma_{b}\left(\varrho_{2}, \mathcal{R} \varrho_{2}\right), \frac{\sigma_{b}\left(\varrho_{1}, \mathcal{R} \varrho_{2}\right)+\sigma_{b}\left(\varrho_{2}, \mathcal{R} \varrho_{1}\right)}{2^{p}}\right\} \\
+L \min \left\{\sigma_{b}\left(\varrho_{1}, \mathcal{R} \varrho_{1}\right), \sigma_{b}\left(\varrho_{2}, \mathcal{R} \varrho_{2}\right), \sigma_{b}\left(\varrho_{1}, \mathcal{R} \varrho_{2}\right), \sigma_{b}\left(\varrho_{2}, \mathcal{R} \varrho_{1}\right)\right\}
\end{array}\right] .}
$$

Taking the function $\Theta \in \Omega_{s}$, we get that (18) is fulfilled. By Theorem 1, we get that the integral inclusion (16) has a solution.

\section{Conclusions}

In this article, we have defined almost multivalued $\left(\Theta, \delta_{b}\right)$-contractions to obtain new fixed point theorems in the setting of complete $b$-metric spaces. As an application of our main theorems, the existence of a solution for a Fredholm integral inclusion is also explored. We hope that the theorems proved in this paper will form new connections for those who are working in $\Theta$-contractions.

Author Contributions: Conceptualization, R.P.A.; Investigation, J.A.; Methodology, J.A.; Project administration, B.A.S.A.; Writing-original draft, J.A.; Writing-review and editing, J.A. All authors have read and agreed to the published version of the manuscript.

Funding: Deanship of Scientific Research (DSR), King Abdulaziz University, Jeddah, Grant No. G: 1433-865-1440.

Acknowledgments: This Project was funded by the Deanship of Scientific Research (DSR) at King Abdulaziz University, Jeddah, under grant No. G: 1433-865-1440. The first author, therefore, acknowledges with thanks DSR for technical and financial support. Authors are grateful to Erdal Karapinar for his comments on the first dfraft of our paper.

Conflicts of Interest: The authors declare no conflict of interest.

\section{References}

1. Bakhtin, I.A. The contraction mapping principle in almost metric spaces. Funct. Anal. 1989, 30, $26-37$.

2. Czerwik, S. Contraction mappings in b-metric spaces. Acta Math. Inform. Univ. Ostra. 1993, 1, 5-11.

3. Czerwik, S.; Dlutek, K.; Singh, S.L. Round-off stability of iteration procedures for operators in $b$-metric spaces. J. Nat. Phys. Sci. 1997, 11, 87-94.

4. Czerwik, S. Nonlinear set-valued contraction mappings in b-metric spaces. Atti Semin. Mat. Fis. Univ. Modena 1998, 46, 263-276.

5. Karapınar, E.; Noorwali, M. Dragomir and Gosa type inequalities on b-metric spaces. J. Inequal. Appl. 2019, 2019, 29. [CrossRef]

6. Ćirić, L.B. Fixed points for generalized multivalued contractions. Mat. Vesnik 1972, 9, 265-272. 
7. Khan, M.S.; Cho, Y.J.; Park, W.T.; Mumtaz, T. Coincidence and common fixed points of hybrid contractions. J. Aust. Math. Soc. 1993, 55, 369-385. [CrossRef]

8. Rhoades, B.E.; Singh, S.L.; Kulshrestha, C. Coincidence theorems for some multi-valued mappings. Int. J. Math. Math. Sci. 1984, 7, 429-434. [CrossRef]

9. Jleli, M.; Samet, B. A new generalization of the Banach contraction principle. J. Inequal. Appl. 2014, $2014,38$. [CrossRef]

10. Jleli, M.; Karapinar, E.; Samet, B. Further generalizations of the Banach contraction principle. J. Inequal. Appl. 2014, 2014, 439. [CrossRef]

11. Li, Z.; Jiang, S. Fixed point theorems of JS-quasi-contractions. Fixed Point Theory Appl. 2016, $2016,40$. [CrossRef]

12. Cosentino, M.; Jleli, M.; Samet, B.; Vetro, C. Solvability of integrodifferential problems via fixed point theory in $b$-metric spaces. Fixed Point Theory Appl. 2015, 2015, 70. [CrossRef]

13. Hussain, N.; Al-Mazrooei, A.E.; Ahmad, J. Fixed point results for generalized $(\alpha-\eta)-\Theta$ contractions with applications. J. Nonlinear Sci. Appl. 2017, 10, 4197-4208. [CrossRef]

14. Hussain, N.; Ahmad, J.; Ćirić, L.B.; Azam, A. Coincidence point theorems for generalized contractions with application to integral equations. Fixed Point Theory Appl. 2015, 2015, 78. [CrossRef]

15. Hussain, N.; Parvaneh, V.; Samet. B.; Vetro, C. Some fixed point theorems for generalized contractive mappings in complete metric spaces. Fixed Point Theory Appl. 2015, 2015, 185. [CrossRef]

16. Hussain, N.; Ahmad, J.; Azam, A. On Suzuki-Wardowski type fixed point theorems. J. Nonlinear Sci. Appl. 2015, 8, 1095-1111. [CrossRef]

17. Ahmad, J.; Hussain, N.; Khan, A.R.; Azam, A. Fixed Point Results for Generalized Multi-valued Contractions. J. Nonlinear Sci. Appl. 2015, 8, 909-918. [CrossRef]

18. Ahmad, J.; Al-Mazrooei, A.E.; Cho, Y.J.; Yang, Y.O. Fixed point results for generalized $\Theta$-contractions. J. Nonlinear Sci. Appl. 2017, 10, 2350-2358. [CrossRef]

19. Al-Rawashdeh, A.; Ahmad, J. Common Fixed Point Theorems for JS-Contractions. Bull. Math. Anal. Appl. 2016, 8, 12-22.

(C) 2020 by the authors. Licensee MDPI, Basel, Switzerland. This article is an open access article distributed under the terms and conditions of the Creative Commons Attribution (CC BY) license (http://creativecommons.org/licenses/by/4.0/). 